\title{
Role, function and challenges of multidisciplinary centres for rare diseases exemplified for neurofibromatosis type 1 syndrome
}

\author{
Hagit Toledano-Alhadef ${ }^{1,2,3}$ (D) Victor-Felix Mautner ${ }^{4}$ Isabel Gugel ${ }^{5,6} \cdot$ Julian Zipfel $^{5,6,7} \cdot$ Karin Haas-Lude $^{5,8}$. \\ Shlomi Constantini ${ }^{1,3,9} \cdot$ Martin U. Schuhmann ${ }^{5,6,7}$
}

Received: 13 May 2020 / Accepted: 26 May 2020 / Published online: 8 June 2020

(C) Springer-Verlag GmbH Germany, part of Springer Nature 2020

\begin{abstract}
Purpose Neurofibromatosis type $1(\mathrm{NF} 1)$ syndrome is a common rare/orphan disease that manifests itself early in the paediatric age. It imposes a considerable burden upon patients as well as on caregivers. Decisions regarding optimal care often rely on several medical instances working together as a team.

Methods The authors reviewed the literature and supplied a description of their own clinical work at the NF1 centres.

Results The experience of a multidisciplinary teamwork of three NF centres was summarized in order to enhance awareness for possible multidisciplinary ways of delivery of health and health-related aspects of care to NF1 patients. Both population-focused research centres and family-focused centres were reviewed.

Conclusions Chronic rare diseases that start in the paediatric age mandate long-term follow-up most often by several disciplines. NF1 syndrome is an example of a multidisciplinary centre in order to enhance the quality of care.
\end{abstract}

Keywords Neurofibromatosis type $1 \cdot$ Orphan disease $\cdot$ Multidisciplinary Team

\section{Introduction}

Neurofibromatosis type 1 (NF1) syndrome is the classical model of a chronic and complex genetically determined condition necessitating a life-long multidisciplinary healthcare approach. It is, although a rare disease according to the definition, a relatively common inherited disorder occurring with a birth incidence of approximately one in 2500-3000 individuals [1]. It is caused by an autosomal dominant mutation in the neurofibromin gene located on chromosome 17 [2].

Shlomi Constantini

sconsts@netvision.net.il

1 Gilbert Israeli and International Neurofibromatosis Centre, Dana-Dwek Children's Hospital, Tel Aviv Sourasky Medical Centre, Tel Aviv, Israel

2 Paediatric Neurology and Child Development Center, Dana-Dwek Children's Hospital, Tel Aviv Sourasky Medical Centre, Tel Aviv, Israel

3 Sackler Faculty of Medicine, Tel Aviv University, Tel Aviv, Israel

4 International Neurofibromatosis Centre, Department of Neurology, University Hospital Hamburg Eppendorf, Hamburg, Germany
Neurofibromatosis type 2 (birth incidence of approximately one in 25,000) [3,4] and the relatively recently established 'young' entity schwannomatosis (with a prevalence of $1: 126,000)$ [4] are rarer, although schwannomatosis in particular is suspected to have a high number of unreported cases with a frequently oligosymptomatic course, which is also reflected in the rising incidence figures.

This paper suggests the NF1 syndrome to serve as an example of a complex multidimensional paediatric rare disease and elaborates on the challenges posed by the necessary multidisciplinary approach with regard to diagnostics, clinical management and research.

5 Centre of Neurofibromatosis at the Centre of Rare Diseases, Tuebingen University Hospital, Tuebingen, Germany

6 Department of Neurosurgery, Tuebingen University Hospital, Tuebingen, Germany

7 Division of Paediatric Neurosurgery, Department of Neurosurgery, Tuebingen University Hospital, Tuebingen, Germany

8 Department of Paediatric Neurology and Developmental Medicine, University Children's Hospital, Tuebingen, Germany

9 Department of Paediatric Neurosurgery, Tel Aviv Sourasky Medical Centre, Tel Aviv, Israel 


\section{Methods}

The authors reviewed the literature concerning the multiple aspects of multidisciplinary clinics and teamwork. They then supplied a description of the existing solutions and approaches in their NF centres and raised issues and suggestions in an effort to set out and determine what could be a better way of applying and conveying the standard of care that should be recommended for a tertiary Multidisciplinary Team clinic (MDT) in NF1 syndrome.

\section{Results}

\section{Demands for a neurofibromatosis (NF1) centre}

NF1 syndrome, classically seen as a genetically driven tumour predisposition syndrome of the central and peripheral nervous system, is in fact a complex multi-system disease that leads, apart from the formation of tumours, to many other impairments, including cognition, bone metabolism, endocrinology and skin affection, and requires intense interdisciplinary cooperation between clinical physicians and scientists from many disciplines (e.g. neurology, dermatology, several surgical disciplines, paediatrics, psychology, neuroradiology, ophthalmology, ear, nose, throat (ENT).

This wide variety of manifestations $[5,6]$ results in immense importance and demand for multidisciplinary treatment and follow-up. Children with NF1 and quite often also some members of their family need regular medical follow-up with regard to all specific manifestations of the disease, thus necessitating multiple healthcare professionals' attention.

In chronic diseases, clinical as well as self-well-being considerations should be addressed simultaneously. Observations from medical fields exist, e.g. the 'Rural Australian Diabetes Inspiring Control Activity \& Lifestyle' model [7]. This model, called 'RADICAL' comprised of a locally based, co-located core team of a general paediatrician, diabetes educator and mental health nurse. Consultation behaviour, including personality expression and team interaction, was an important part of the success of the RADICAL model. The paediatrician no longer carried the sole burden of management, leading to a change in consultation behaviour. [7].

In the authors opinion, the following demands should be met by a centre for neurofibromatosis (and other centres for rare diseases):

- Establishment of a treatment and research centre for neurofibromatosis as a coordinating lead centre.

- Implementation of diagnostic and therapeutic standards for the qualitative diagnosis and treatment of neurofibromatosis.
- Development of new therapy options through interdisciplinary networking of clinic and research.

- Intensification of basic research through the promotion of interdisciplinary and international cooperation and through the acquisition of external funding.

- Further development of education, training and continued education concepts for physicians, nursing staff and students.

- Establishment and expansion of study registries.

- Cooperation with medical societies, self-help groups and other centres for rare diseases.

- Identifying and separating patients with severe clinical course from patients with a mild form of the syndrome. The severe clinical course of the syndrome may include cognitive impairment, severe disfigurement, scoliosis, high internal tumour burden, osteoporosis and osteopenia, which can occur in one patient simultaneously. Such patients need close follow-up in the centre itself. In contrast, patients with mild forms of the disease can be monitored by general practitioners for a long time and are only referred to NF centres at longer intervals.

\section{Experiences in multidisciplinary care centres}

In the following, three models of inter- and multidisciplinary care for children and adolescents (and adults) are described as possible exemplary solutions to meet the named demands for centres. Furthermore, the lessons learnt from the experiences made are discussed, which may differ according to the centre, the society and the local health systems in question.

\section{The experience at the Gilbert Israeli and International Neurofibromatosis Centre}

1. Patients are referred to the GIINFC from all over Israel by paediatricians, paediatric neurologists and adult physicians. The clinic provides multidisciplinary and multispecialized consultations and advanced treatments focused on NF1 syndrome. All clinical appointments with the child and its family take place in a multidisciplinary setting with a social worker and a psychologist attending each formal medical visit.

2. The multidisciplinary team of the GIINFC holds meetings twice a month and includes all the caretakers of adults and children involved such as paediatric and adult neurologists, neurosurgeons, an ophthalmologist, orthopaedics, oncologists, a geneticist and a radiologist. Other specialists are added, if needed. This setup enables a continuous flow of information and familiarity with each patient and his family among all the interdisciplinary staff. This physical proximity of multiple professionals under one roof in 
one hospital segment enables smoother and more accurate communication and delivery of care to the patient and his family.

3. The GIINFC provides greater access to families who have several members with NF1 syndrome to the multidisciplinary team by enabling single day coordinated visits, both by paediatric and adult neurologists.

4. The GIINFC includes children and adult clinics under the same roof. This enables the team to provide comprehensive and continuous care to children ( $<18$ years) and adults ( $>18$ years) implementing a unique model of transition for adolescents and young adults. This setup allows a smooth transition from the paediatric clinic to the adult section, as the young adult moves from the paediatric to the adult neurologist, with no changes among the rest of the staff, and thus avoiding gaps of medical care.

5. The patient has one point of contact, is accompanied by the social worker and the psychologist throughout the whole treatment and receives emotional support in case of difficulties. At the same time, the family of the adolescent gradually redefines its role in the caretaking process.

6. Clinical research has focused on optic pathway gliomas, imaging nuances including volumetrics, cognitive aspects and psychological implications.

\section{The experience at the Centre of Neurofibromatoses at the Centre of Rare Diseases, University Hospital and Eberhard Karls University Tübingen, Germany}

1. The Centre of Rare Diseases in Tübingen was founded in 2010 as Germany's first Centre of Rare Diseases. It comprises currently of 14 sub-centres, one of which is the Centre of Neurofibromatoses (CNF). The CNF is run by the following core members: Departments of Neurosurgery (including Division of Paediatric Neurosurgery), Paediatric Neurology and Developmental Medicine, Dermatology, and Institute of Human Genetics. Official cooperation has been established with fifteen diagnostic, surgical and conservative departments. The centre sees patients with NF1, NF2, schwannomatosis and associated rasopathies from all over Germany and some international patients.

2. The CNF offers weekly parallel clinics for NF1 adults and NF1 children/adolescents so that all NF1 family members can be seen on the same day. The majority of children/ adolescents have non-surgical issues and are primarily seen in Paediatric Neurology; children referred for surgical issues are primarily seen by Paediatric Neurosurgery. If other clinical issues are detected, the child is transferred to the respective clinic under the same roof.

3. Paediatric Neurology runs further clinics for NF1 patients with special needs, offering multidisciplinary age-specific monitoring of the associated clinical manifestations. This includes a focus on developmental disorders. There is close cooperation with the nationwide 'Social Paediatric Centres', in which patients with deficits are cared for close to their homes.

4. All adults with NF1 and NF2 are seen by Neurosurgery. Children/adolescents with NF2 are primarily seen by Paediatric Neurosurgery in parallel to the adult NF clinic.

5. Recently, the state of Baden Württemberg recognized the Centre of Rare Diseases (CRD) as part of the state public health program. A term of the negotiation requires health insurances to pay for the special care of CRD patients beyond standard needs. This enabled the hiring of two nurse practitioners taking care of the nonsurgical needs of all adults, including work and psychological issues, communications with authorities, networking and mentoring for daily life issues. The program includes the organization of the transition from the paediatric hospital and the social paediatric centres to the adult world.

6. A current project is the establishment of interdisciplinary case conferences with video streaming to enable other hospitals and practitioners to present and discuss their cases.

7. Clinical research has focused on NF2 and hearing preservation and on cognition and attention-deficit/hyperactivity syndrome in NF1.

\section{The Hamburg International NF Centre experience}

- The ICNF in Hamburg was established in 1991. The core consists of a stand-alone outpatient clinic recognized and financed by the German Public Health System. Recently, medical care has also been provided in the form of video consultations. The outpatient clinic sees about 1000 patients per year, both adults and children, with all forms of neurofibromatosis and rasopathies. The centre sees patients from all over Germany and international patients.

- The majority of patients come to the outpatient clinic approximately once a year. Here most of the routine diagnostics (electrophysiology, neuropsychological assessment, consultations with other specialities) and coordination of further treatment takes place. In addition, patients receive genetic counselling and local contact points are arranged.

- In cooperation with an external radiological institute in Hamburg, a NF-specific, comprehensive, time-optimized, high-quality routine radiological diagnostics on a budgetneutral basis including whole-body MRI and highresolution special imaging has been established.

- In the associated NF research laboratory of the Hamburg University Medical Centre, all necessary molecular genetic examination methods (marker analysis, mutation 
analysis, etc.) are established. At the same time, new diagnostic methods are being tested and further developed.

- A close cooperation has developed with numerous departments of the Hamburg University Medical Centre. Special psychiatric and neuropsychological diagnostics are offered to patients with psychological and cognitive stress factors.

- The Foundation of the German National NF patient lay organization 'Bundesverband Neurofibromatose' was initiated, supported and counselled by the ICNF. Its headquarter in Bonn plays an enormous role in all non-clinical aspects of life for patients with NF, especially with respect to counselling and information brochures.

- Clinical research has a long tradition in the ICNF with over 200 publications. Studies have focused on plexiform neurofibromas (PNF) and malignant peripheral nerve sheath tumours (MPNST) to clarify the biology of these tumours. For the first time, the volumetry of PNF could identify patient groups with increased risk for MPNST. The bone metabolism of NF1 patients was investigated in detail. Recently, the risk and survival of women with contralateral breast cancer in NF1 were described in a European collaboration. In cooperation with ENT, ophthalmology and oncology, the first treatments of NF2 patients in Germany with the angiogenesis inhibitor Bevacizumab were initiated within the framework of individual treatment trials.

\section{Discussion}

Chronic diseases mandate long-term follow-up often by several disciplines, both medical and non-medical. There is consensus among headache specialists, for example that a multidisciplinary treatment/team approach to treating refractory headaches has advantages and results in better outcomes [8]. The multidisciplinary model of care is defined as a range of healthcare professionals working independently, but in a coordinated manner to improve outcomes especially for chronic diseases [9].

Rare diseases are increasingly recognized as the orphans of medicine and have lately received special attention in Western societies. NF1 syndrome is a perfect example of the multidimensional aspects from birth to late adulthood due to the complexity of many possibly affected systems, the very individual disease burden even in genetically identical twins [10-13] and within families, and finally the limited knowledge of the disease specificities by general practitioners and paediatricians. Specialists in private practices or in hospitals almost always only see 'their' part of the disease according to their specialization, but not the whole picture.
A recent report has stressed the importance of MDT work in neurocutaneous syndromes, and especially in NF1 syndrome, and emphasized the challenges of close surveillance of potential clinical complications even in the given multidisciplinary outpatient clinic [14].

Focusing patients in centres, which can have all kinds of different organizational forms as outlined by the three examples described by the authors of this manuscript, seems mandatory for coping with as many aspects of the disease as possible. None of the three examples is perfect and all organizational forms have their shortcomings and their individual history of development in their given environment. They are, however, similar in their bottom-line approach: primarily, to be open to all affected patients and families to describe and identify the individual disease burden. Secondarily, in case a complex manifestation exists, to address it with the power of an organizational network that should not only cover the patient but also his family and social network.

Regarding the care of children and adolescents, the familycentred care approach (FCCA) is widely used in healthcare centres and recognized as an approach that can improve the effectiveness and efficiency of health services, in particular in paediatric rehabilitation centres [15]. In different chronic conditions, there is a growing understanding that a whole family approach should be implemented in order to address all the needs of the patient and the family. The provision of familycentred services emphasizes a partnership between parents and service providers so that families are involved in every aspect of services for their child [16].

Regarding clinical research in rare diseases, only the concentration of patients in centres allows access to larger cohorts of patients. Furthermore, academic affiliation offers a better opportunity to concentrate the expertise of university hospitals facilities and research institutions on rare diseases and to overcome the lack of public interest in a rare disease problem, especially if other, more obvious health issues of society are still not being sufficiently approached. Furthermore, rare diseases mandate national and international cooperation for clinical and research projects, as exemplified by the European Reference Networks (ERN) for NF, e.g. the GENTURIS network for rare genetic tumour risk syndromes (www.genturis.eu).

On the other hand, it seems reasonable that NF1 centres should dichotomize patients into a high disease burden and a complex disease pattern group, and a group manifesting milder aspects of the syndrome. The first group needs regular visits and full attention of centre opportunities and possibilities, while for the second, the centre should provide the primary caregivers with a written guideline for the necessary items of disease monitoring and a list of unnecessary investigations, for example yearly routine head MRI scans in children.

NF1 centres thus carry the responsibility for teaching general practitioners, neurologists, paediatricians, dermatologists, 
specialists in private practice and in hospitals alike, with regard to the needs and requirements of a rare disease like NF1 syndrome.

This can be done, for example by establishing national guidelines and standard operating procedures (SOPs) for diagnosis and treatment as recently demonstrated by the French National Guidelines for NF1 syndrome [17], an excellent example made available to a whole country but also to other nations and centres.

Academies for teaching colleagues in a clinical setting need also to reach out to the education of patients and parents, in their language regarding their issues and needs. In the past, the focus has been on regional or national meetings for both groups, still leaving much room for improvement in most of our societies.

Given that MDT meetings utilize considerable time, effort and financial resources $[18,19]$, emphasis should be placed on whether there is a need for quantifying the value of a multidisciplinary clinic. It is essential to recognize the importance of enhanced quality of care provided and the ability to participate in translational research filling the gaps in knowledge. Data available regarding integrated healthcare models suggest improved treatment outcomes and reduced healthcare costs [20]. To date, these models include primary care physicians and behavioral health specialists working together often on a referral basis. These models, however, fall short in real-time coordination of care in which specialists see the patient during the same clinic visit [21].

Several reports have described the MTD clinics before [22] and tried to outline the structure and function of such clinics in order to improve patient outcomes, but significant heterogeneity exists in such clinics with regard to team composition, entry criteria, follow-up and a variability of processes. Therefore, this work is in need of standardization.

In addition, the SARS-CoV-2 pandemic has taught us that digital education formats like webinars, video conferences and live streams will need to be developed to continue and possibly enhance the educational efforts for improving the care of NF1 syndrome and other rare diseases. The power of digital education and data conveying has to be strapped-on to the future needs of working remotely.

This special issue of the Child's Nervous System is another attempt to improve the care of children with phacomatoses by providing a comprehensive collection of educative reviews and research papers.

Acknowledgements The authors would like to thank Monique Ben-Am for editorial assistance.

\section{Compliance with ethical standards}

Conflict of interest On behalf of all authors, the corresponding author states that there is no conflict of interest.

\section{References}

1. Lammert M, Friedman JM, Kluwe L, Mautner VF (2005) Prevalence of neurofibromatosis 1 in German children at elementary school enrollment. Arch Dermatol 141:71-74. https://doi.org/ 10.1001/archderm.141.1.71

2. Ledbetter DH, Rich DC, O’Connell P, Leppert M, Carey JC (1989) Precise localization of NF1 to $17 \mathrm{q} 11.2$ by balanced translocation. Am J Hum Genet 44:20-24

3. Evans DGR, Moran A, King A, Saeed S, Gurusinghe N, Ramsden $R$ (2005) Incidence of vestibular schwannoma and neurofibromatosis 2 in the North West of England over a 10-year period: higher incidence than previously thought. Otol Neurotol 26:93-97. https:// doi.org/10.1097/00129492-200501000-00016

4. Evans DG, Bowers NL, Tobi S, Hartley C, Wallace AJ, King AT et al (2018) Schwannomatosis: a genetic and epidemiological study. J Neurol Neurosurg Psychiatry 89:1215-1219. https://doi. org/10.1136/jnnp-2018-318538

5. Hirbe AC, Gutmann DH (2014) Neurofibromatosis type 1: a multidisciplinary approach to care. Lancet Neurol 13:834-843. https:// doi.org/10.1016/S1474-4422(14)70063-8

6. Ferner RE, Huson SM, Thomas N, Moss C, Willshaw H, Evans DG et al (2007) Guidelines for the diagnosis and management of individuals with neurofibromatosis 1 . J Med Genet 44:81-88 https:// doi.org/10.1136/jmg.2006.045906

7. Pw G, Paterson MA, Renalson JA (2010) A ' radical ' new rural model for pediatric diabetes care. Pediatr Diabetes 11:296-304. https://doi.org/10.1111/j.1399-5448.2009.00594.x

8. Sahai-Srivastava S, Sigman E, Uyeshiro Simon A, Cleary L, Ginoza L (2017) Multidisciplinary team treatment approaches to chronic daily headaches. Headache 57:1482-1491. https://doi.org/ 10.1111/head.13118

9. Wagner EH (2000) The role of patient care teams in chronic disease management. Br Med J 320:569-572. https://doi.org/10.1136/bmj. 320.7234.569

10. Sites ER, Smolarek TA, Martin LJ, Viskochil DH, Stevenson DA, Ullrich NJ et al (2017) Analysis of copy number variants in 11 pairs of monozygotic twins with neurofibromatosis type 1 . Am J Med Genet Part A 173:647-653. https://doi.org/10.1002/ajmg.a.38058

11. Payne MS, Nadell JM, Lacassie Y, Tilton AH (2003) Congenital glaucoma and neurofibromatosis in a monozygotic twin: case report and review of literature. J Child Neurol 18:504-508. https://doi.org/ 10.1177/08830738030180071101

12. Sabbagh A, Pasmant E, Laurendeau I, Parfait B, Barbarot S, Guillot B et al (2009) Unravelling the genetic basis of variable clinical expression in neurofibromatosis 1. Hum Mol Genet 18:27682778. https://doi.org/10.1093/hmg/ddp212

13. Rieley MB, Stevenson DA, Viskochil DH, Tinkle BT, Martin LJ, Schorry EK (2011) Variable expression of neurofibromatosis 1 in monozygotic twins. Am J Med Genet Part A 155:478-485. https:// doi.org/10.1002/ajmg.a.33851

14. Kokkinou E, Roka K, Alexopoulos A, Tsina E, Nikas I, Krallis P et al (2019) Development of a multidisciplinary clinic of neurofibromatosis type 1 and other neurocutaneous disorders in Greece. A 3-year experience. Postgrad Med 131:445-452. https://doi.org/10. 1080/00325481.2019.1659708

15. Lavigne J, Rushton PW, Trudelle N (2017) Perceptions of a multidisciplinary team regarding a pediatric rehabilitation modified needs assessment. Scand J Occup Ther 24:431-437. https://doi. org/10.1080/11038128.2017.1320584

16. Law M, Hanna S, King G, Hurley P, King S, Kertoy M et al (2003) Factors affecting family-centred service delivery for children with disabilities. Child Care Health Dev 29:357-366. https://doi.org/10. 1046/j.1365-2214.2003.00351.x 
17. Bergqvist C, Servy A, Valeyrie-Allanore L, Ferkal S, Combemale P, Wolkenstein P et al (2020) Neurofibromatosis 1 French national guidelines based on an extensive literature review since 1966. Orphanet J Rare Dis 15:1-23. https://doi.org/10.1186/s13023020-1310-3

18. Pillay B, Wootten AC, Crowe H, Corcoran N, Tran B, Bowden P et al (2016) The impact of multidisciplinary team meetings on patient assessment, management and outcomes in oncology settings: a systematic review of the literature. Cancer Treat Rev 42:56-72. https://doi.org/10.1016/j.ctrv.2015.11.007

19. Taylor C, Munro AJ, Glynne-Jones R, Griffith C, Trevatt P, Richards $\mathrm{M}$ et al (2010) Multidisciplinary team working in cancer: what is the evidence? BMJ 340:743-745. https://doi.org/10.1136/ bmj.c951

20. Unützer J, Katon W, Callahan CM, Williams JW, Hunkeler E, Harpole L et al (2002) Collaborative care management of late-life depression in the primary care setting: a randomized controlled trial. J Am Med Assoc 288:2836-2845. https://doi.org/10.1001/jama. 288.22.2836

21. Duis J, van Wattum PJ, Scheimann A, Salehi P, Brokamp E, Fairbrother L et al (2019) A multidisciplinary approach to the clinical management of Prader-Willi syndrome. Mol Genet Genomic Med 7:1-21. https://doi.org/10.1002/mgg3.514

22. Collister D, Pyne L, J, Donald M, Molnar A, Beaulieu M et al (2019) Multidisciplinary chronic kidney disease clinic practices: a scoping review. Can J Kidney Heal Dis 6. https://doi.org/10.1177/ 2054358119882667

Publisher's note Springer Nature remains neutral with regard to jurisdictional claims in published maps and institutional affiliations. 